\title{
Teaching dynamics in engineering technology through software tools
}

\author{
Ratan Kumar \\ Department of Engineering Technology \\ University of North Texas, Denton, TX 76203
}

\begin{abstract}
The use of software as a teaching aid is rapidly gaining popularity. This paper describes some of the software available that helps in teaching dynamics to students of engineering technology. The instructional emphasis in engineering technology is application based. Problems and assignments are given that helps to illustrate the theory from a practical perspective and the stress on theory itself is less. Modern educational tools like simulation and multimedia help the instructor to exemplify theory through industrial examples that are sometimes difficult to describe in textbooks and easier to comprehend through visualization. This technique is also well recieved by students who gain more insight to the theory and feel less burdened in overcoming the associated mathematical difficulties.
\end{abstract}

\section{Introduction}

Engineering technology and engineering programs have dispareted themselves in that the former places more emphasis on the application of theory. This helps the students to learn in a manner that can be immediately translated towards industrial applications. Dynamics is quite often one of the first technical courses encountered by engineering technology students. It sets up the stage for the instructors to teach the applied aspect of science and engineering. In order to elucidate the theory, application oriented problems have to be provided. Although the current textbooks contain a plethora of such problems the visualization and simulation process provides a more efficient means in discerning the theory. Dynamics is a subject that depends on geometric and physical perception and every effort should be made to enhance this ability. Through modern processes the instructors, in engineering technology, can show and assign a multitude of problems by a pseudo-live technique and spend less time in showing the mathematical intricasies. This method also helps the students to get exposed and exploit the state of the art software available for engineering practices.

The course was designed around real world problems. In order to understand the subject as an engineer, theory and problem solving strategies were covered in the lecture period. The objective was to teach the students, how to approach problems and critically judge the results. Simulation and multimedia tools were used to animate the problems and provide a real world effect to them. Visualization was afforded through models, pictures, graphs and simulation. The use of these tools helped to supplement the class lecture. Homework assignments were computer based and it was given keeping in mind the use of software tools. The use of mathematical tools was encouraged to perform repetitious calculations. This helped the students to spend more time in understanding the problem and less time in solving various forms of it. Also some prebuilt simulation packages were provided so that the students could run them and solve a problem from different perspectives. Projects were given which emphasized design issues. This helped the students in understanding the associated engineering issues. 


\section{Software usage}

A variety of software was used during classroom lecture and for homework assignments. These software helped in multimedia application, providing simulation, performing mathematical manipulations and spreadsheet analysis. Previously created models were often used for software that needed an extra learning time. The multimedia tool as a learning aid holds a lot of promise in providing a good learning mechanism. This helps the students to learn by acting and reflecting and not by watching and listening. Currently most of the multimedia tools are on CD ROM's. However in the area of dynamics, not many are available. The author used the work by Grammoll' extensively. Figure 1 shows a sample screen availble in the software for

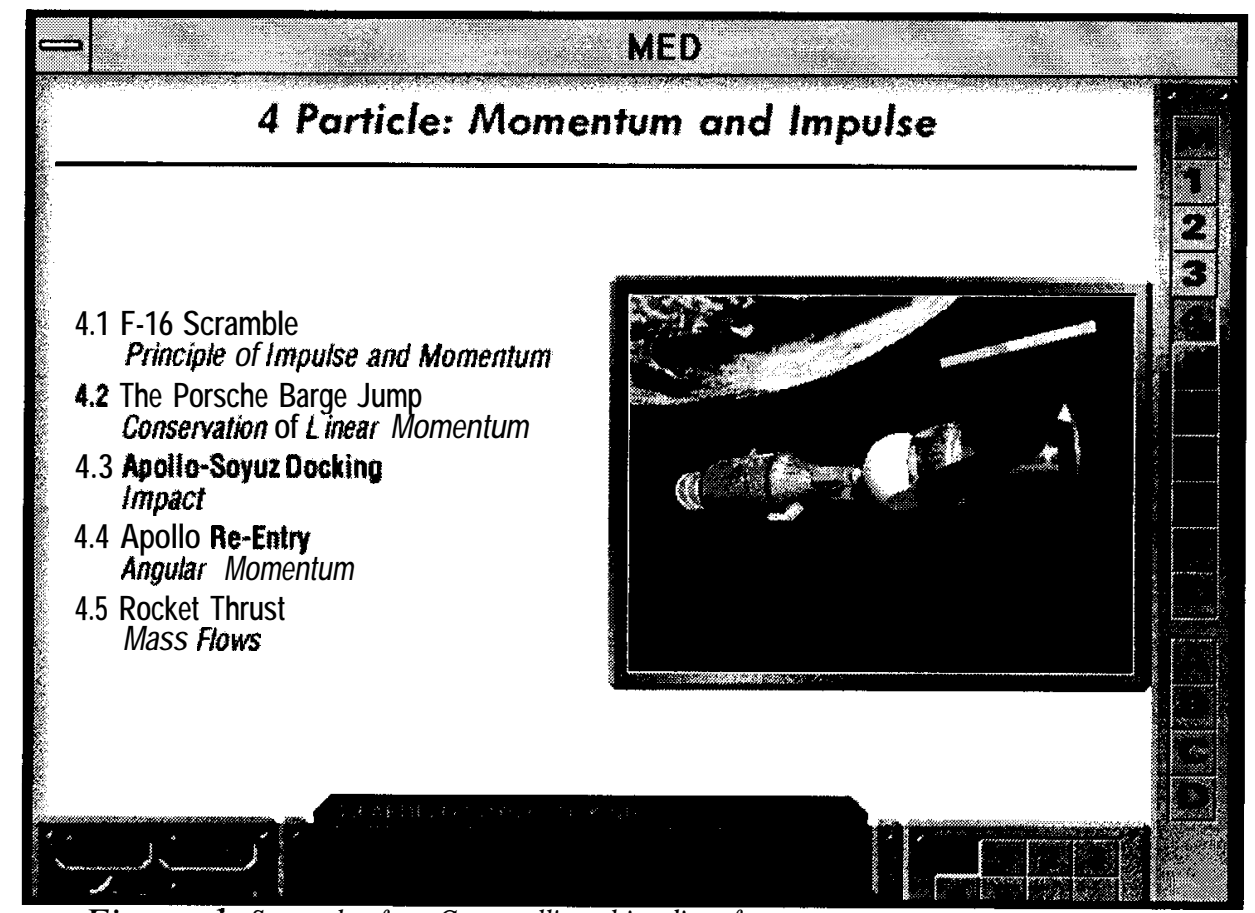

Figure 1. Screenshot from Grammoll's multimedia software

interaction purpose. The software contains about forty three real-world problems which cover a typical dynamics course. It is divided up into ten sections each of which has at least two illustrative problems explaining a particular theory. For example, Figure 1 is the introductory screen for one of the sections and it has five problems. Each problem is discussed by an introductory section, the associated theory and problem definition, the solution and a simulation. They are presented by text, graphics, animations and audio. The simulation software gives user the power to dynamically manipulate the physical system and explore the engineering concepts. Figure 2 shows the simulation screen for problem three as shown in Figure 1.

The simulations used for coursework purpose were developed in Working $\operatorname{Model}^{2}$ from The Knowledge Revolution. It is a motion simulation package that allows one to quickly build and analyze dynamic mechanical systems on desktop computers. Most of the exmples that were shown by this simulation package were pre-built. This not only saved time but building models was deemed unnecessary since several such models already exist. Bedford and Fowler ${ }^{3}$ have 


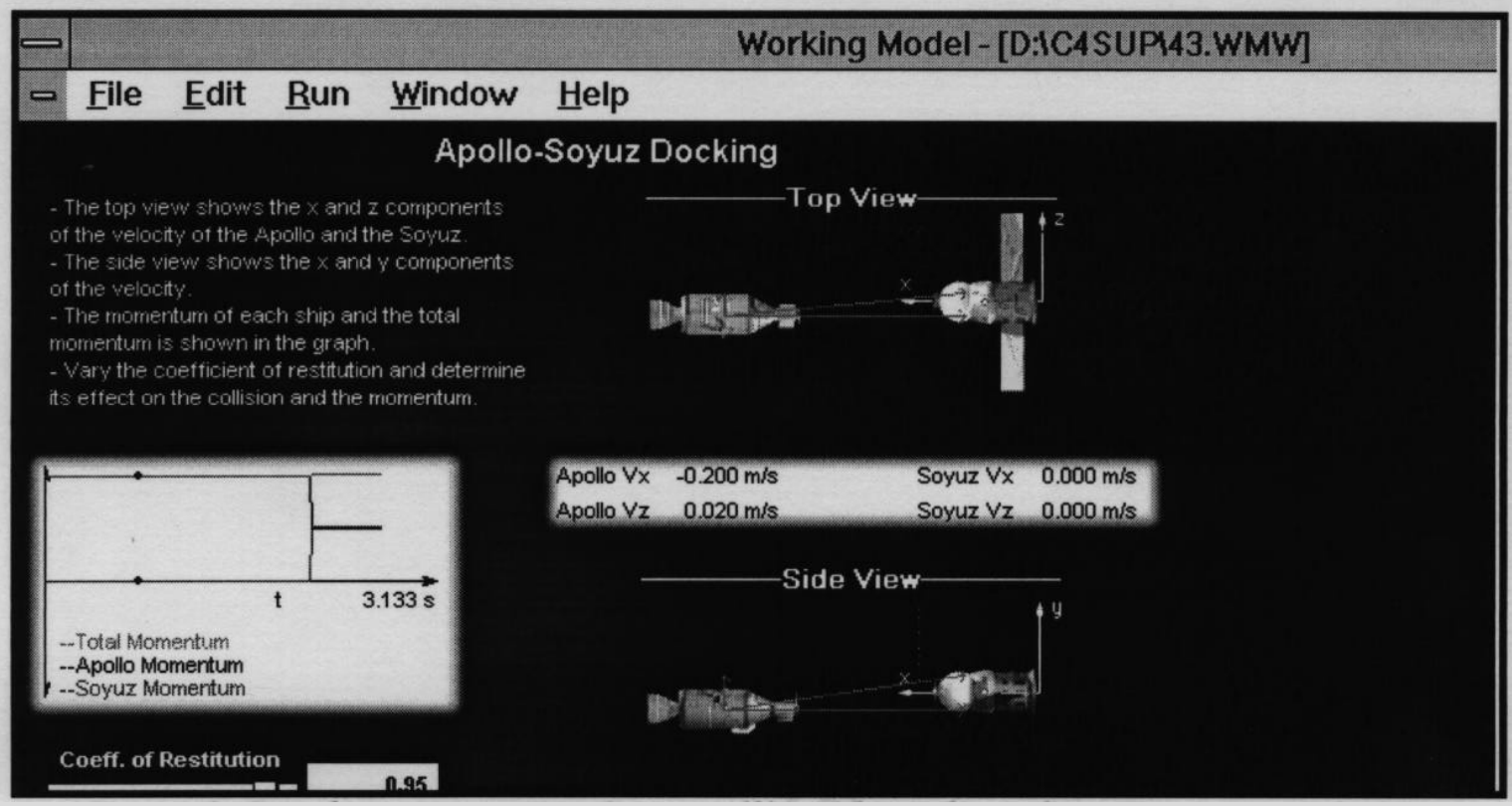

Figure 2. Simulation screen in Grammoll 's multimedia software

around hundred problems and examples in their textbook. They have been recreated on a disk as Working Model simulations and can be used in a variety of situations. Figure 3 shows a situation were design issues like backlash in gears can be detected. This simulation was created by Knowledge Revolution themselves. The main purpose was to expose the students to such

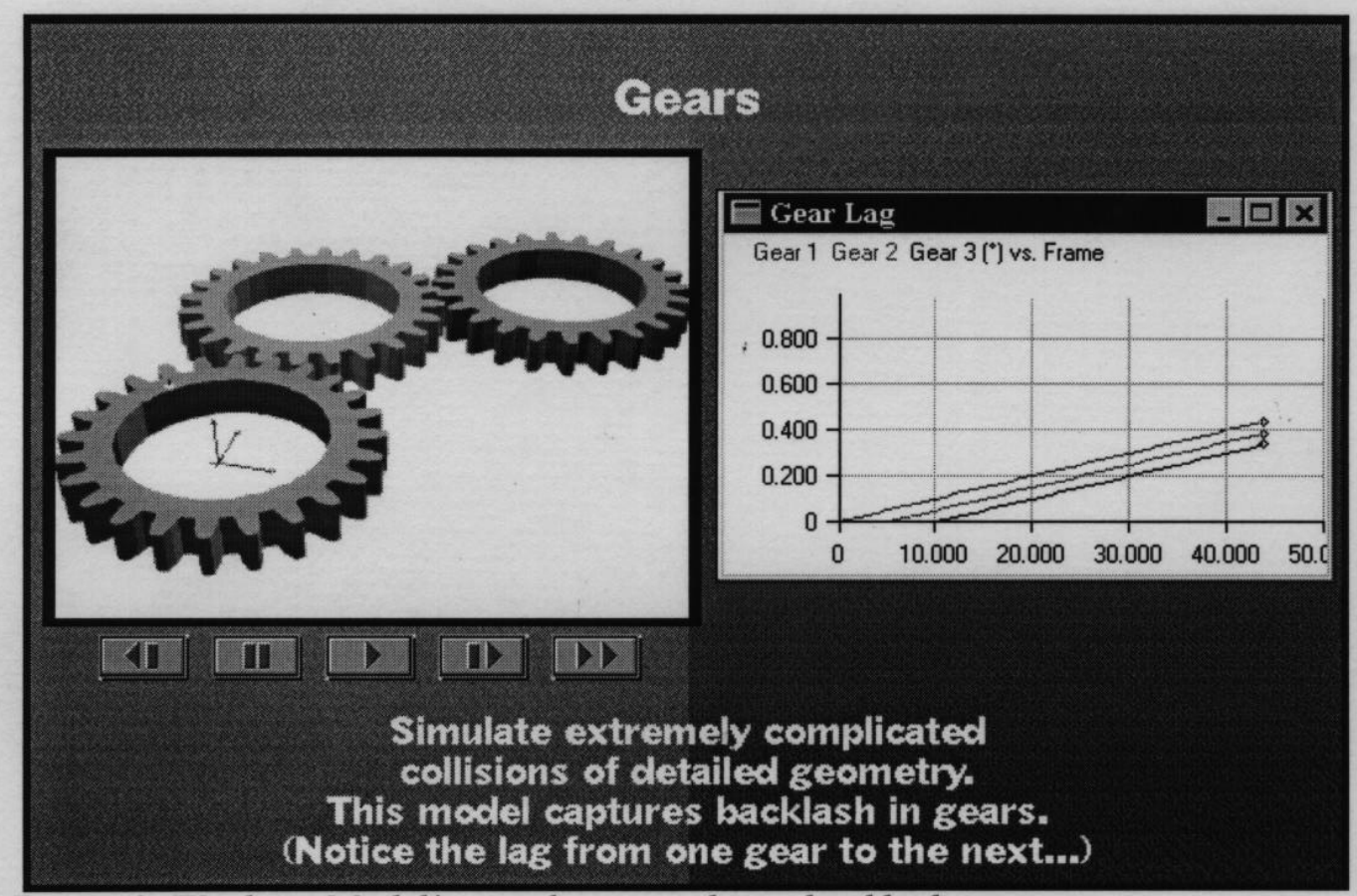

Figure 3. Working Model's simulation to detect backlash in gears. 
packages which are later used in senior classes. With a similar intent an example from a finiteelement software package Algor ${ }^{4}$ was also used. The use of the finite-element package was cursory as it can confuse the students at an early engineering stage. However some examples shown were calculation of weight, center of gravity and mass moment of inertia of plates. The problems were selected to show how numerical analysis can very accurately match theoretical results. Several such case studies can be accessed on Algor's world-wide-web page.

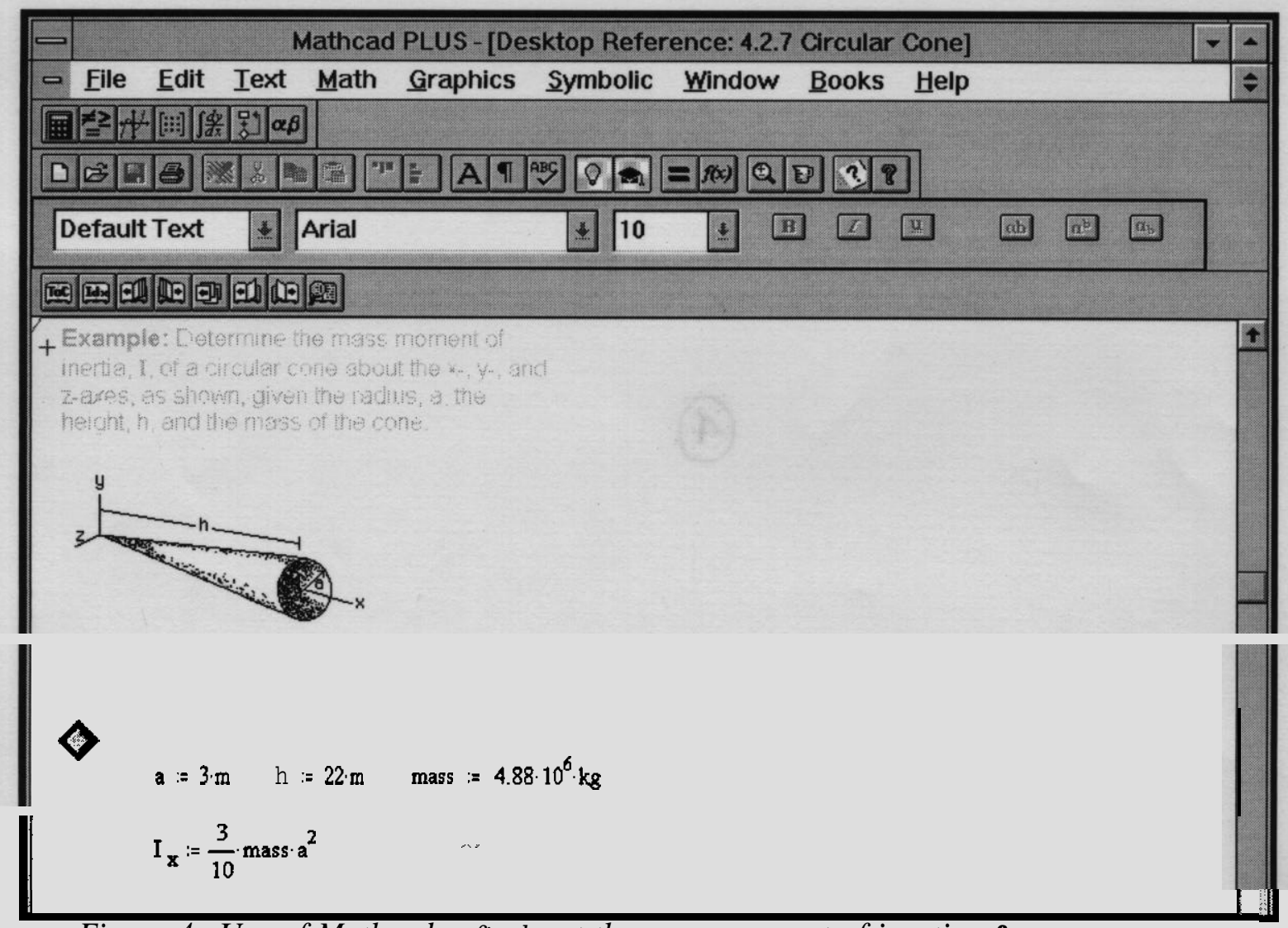

Figure 4. Use of Mathcad to find out the mass moment of inertia of a cone

The use of mathematical software was highly encouraged so that the students did not get constrained with mathematical manipulations but learned the subject matter. Mathcad was chosen as the candidate for this purpose. The choice was dictated by the availability of this software to our students and it should be noted that several competitive packages are available in this category. These packages help to put text, graphics, equations and numbers all on one sheet as is seen in a textbook. However they are interactive in that they can solve equations and perform other mathematical activities. The equations and graphs are live and a change in data redraws the graph. Some other features that make it attractive is that it can track standard units, calculate properties like moment of inertia through its built in functions. Figure 4 shows how mass moment of inertia of a cone can be computed and it is to be noted that as soon as one of the parameters is changed the updated answer is immediately posted. Some use of spreadsheets like Excel $^{6}$ was also employed throughout the course. Spreadsheets are becoming increasingly powerful and are an important tool used in industry, we found it useful to make the students proficient at them. 


\section{Conclusion}

The use of application software as a supplemental tool to teach dynamics was found very useful. The Engineering Technology program requires that the students be exposed to industrial situations most of the time through problems and examples. Although such illustrations are available in many textbooks, the use of multimedia and simulation tools to exemplify them is beneficial. The use of such software helps the students to visually comprehend dynamic systems and complicated motion in a better way. Since the aim of the class was to teach students the subject of dynamics and not let them get caught up with mathematical juggling, the use of mathematical software was highly encouraged. It was specially favorable when the same mathematical expression had to be solved repeatedly to see a pattern emerge. Class time was appropriately used so that the students get a right mix of theory, explanation, problem solving and multimedia usage. Homework assignments were given so that the students could start thinking like engineers. Design problems were issued as projects to expose students to some real engineering type situations.

\section{References}

[1] Gramoll, K. and Abbanat, R., Multimedia Engineering Dynamics, Addison-Wesley, Reading, MA 1996.

[2] Working Model, The Knowledge Revolution, San Mateo, CA, 1996.

[3] Bedford, A. and Fowler. W., Engineering Mechanics - Dynamics, Addison-Wesley, Reading, MA, 1996.

[4] ALGOR, Algor Inc., Pittsburgh, PA, 1996.

[5] Mathcad, Mathsoft Inc., Cambridge, MA, 1996.

[6] Excel, Microsoft Corporation, Richmond, WA, 1996.

\section{Biographical Information}

RATAN KUMAR obtained a BME in Mechanical Engineering from Jadavpur University (India), ME in Nuclear Engineering from the University of Florida and doctorate in Nuclear/Mechanical Engineering from University of Florida. He has worked as a Mechanical Engineer for three years and since 1992 has taught at American Technical Institute and currently at the University of North Texas. 